\title{
Summary
}

The elbow is a complex joint that is prone to bleeding episodes. These features as well as the close proximity of the ulnar nerve and the need to use the elbow in many activities of daily living can lead to a range of symptoms including recurrent bleeds, pain, instability or loss of range of movement and nerve compression. Conservative management includes splinting and proprioceptive retraining monitored by a musculoskeletal expert in hemophilia care. In the event that conservative measures are not successful a range of surgical options may be indicated including elbow replacement. These approaches continue to be evaluated in both the short and long term in order to determine the most effective treatment for the symptomatic elbow.

\section{Management of the Elbow Joint}

\section{Heim M, Beeton K, Blamey G, Goddard N}

It has been our combined honour to be asked to chair together the orthopaedic session regarding the elbow joint in persons with haemophilia. The elbow is a complex joint involving superior radio-ulnar, humero-ulnar and humero-radial joints. It is prone to bleeding episodes and is cited as the second most commonly affected joint after the knee. This propensity to bleeds, the anatomical and biomechanical complexity, the close proximity of the ulnar nerve and the need to use the elbow in many activities of daily living can lead to a range of symptoms. This includes recurrent bleeds, pain, instability or loss of range of movement and nerve compression. There are many challenges for the management of this joint for both the physiotherapist and orthopaedic surgeon. This paper will provide an overview of the pathophysiology that affects the elbow joint and this will be followed by the conservative management approach. The orthopaedic surgical options will then be presented.

\section{Anatomy and pathophysiology of the elbow joint}

The distal end of the humerus articulates with the proximal end of both the radius and the ulna. In addition there is a joint between the proximal radius and the ulna. The entire complex is contained within a single joint capsule and hence any haemarthrosis will affect all three articulations. The synovial membrane within the joint may hypertrophy resulting in an additional 
flow of nutrients. In young children, this excessive blood flow within the joint capsule enhances growth in the proximal radial epiphysis which may result in hypertrophy of the radial head. This enlarged radial head can be recognized on radiographs and is linked with haemophilia.

The elbow complex of joints allows movement between the upper arm and the forearm. The humero-ulna joint permits extension and flexion of the elbow while the humero-radial and proximal radio-ulna joints allow for pronation and supination of the forearm. The muscles of the elbow joint are known for providing both power, in all directions and for their integrated functioning of pronation and supination during flexion and extension.

In the general population the involvement of the elbow joints is generally related to trauma wherein the joint congruity has been altered. The elbow joint in patients with haemophilia is very different. The application of excessive force through the joint and /or trauma may result in a haemarthrosis. Unless this bleeding episode is dealt with swiftly and the joint returned to its pre bleed condition the bleeding episode may start off a chain reaction comprising synovial hypertrophy, articular cartilage damage, joint shape alteration and intra and extra articular pathologies.

Haemophilia is a systemic disorder of blood coagulation dysfunction and the common joints involved are the knees and ankles. These major joints of the lower limbs function in a compromised way and often the upper limbs are used as auxiliary appendages of ambulation; they are used to pull the patient from a sitting to a standing position or to partially support a patient with lower limb problems by providing support or weight bearing through a cane, crutch or walker frame. This can exacerbate symptoms around the elbow. Haemarthrosis unless efficiently managed contributes to muscle weakness around the joint and hence endangers the joints even further. The enlarged radial head primarily limits supination of the forearm, while extra articular boney changes and muscle fibrosis can produce an entrapment of the ulna nerve which passes the joint in close proximity to the bone. Neurolysis may become necessary. It is important to note that the muscles around the elbow also behave in a different manner to most of the peri-articular muscles in the rest of the body. There is an unexplained tendency for ossification within the muscle substance and hence physiotherapy and rehabilitation programmes suggest the treatment should be gentle and protracted avoiding the use of force. 
Haemophilic arthropathy of the elbow usually begins with hypertrophy of the radial head with resulting impingement on the proximal ulnar facet which ultimately restricts forearm rotation, especially supination. Destructive changes occur insidiously as it is not a classical weight-bearing joint and early limitations of flexion and extension seldom interfere with overall function (1).As the disease progresses to involve the humero-ulnar joint there is progressive restriction of flexion and extension and consequent impairment of normal activities of daily living. Occasionally patients experience an ulnar neuropathy as a result of bone deformity impinging on the ulnar groove.

\section{Conservative management}

Splinting of the elbow joint remains a mainstay of conservative management following or between bleeding episodes. It holds the unique position amidst more invasive approaches of being the only type of management, in theory if not in practice, of being available to all people with hemophilia around the world. The term splinting covers a multitude of applications, each of which realizing their full potential if prescribed, applied, and monitored by a musculoskeletal expert in hemophilia care. Careful and considered selection of the type of device to apply, the wearing schedule, the periodic adjustment of the device itself and the manner in which it is utilized, will maximize the potential benefits to joint and muscle function. Taking into account that a $50 \%$ decrease in elbow motion limits the function of the entire upper extremity by almost $80 \%$ [2], preservation of motion must remain uppermost amongst the goals of treatment, even at times when a period of immobilization may be required. The finding that overuse and disuse of a joint both result in degradation of articular cartilage [3] brings into focus the fact that although there will be times when complete immobilization of a hemophilic elbow is necessary, the length of time that the joint remains fixed in one position should be carefully monitored and restricted to only the absolute minimum therapeutic duration. Similarly, joints that require structural support in order to maintain more normal kinematic patterns must be recognized and the appropriate orthosis applied to mitigate tissue injury from active mobilization. 
In addition to considering the structural integrity of the joint, clinicians must address the proprioceptive capabilities and responsibilities of the elbow as they relate to hand and upper limb function. The ability to perform well-trained reaching movements depends on coordinated sensory input and motor output cooperation. Some authors have suggested that the availability of visual information plays a minor role in this process, and that proprioceptive information is the main feedback source working to control these movements [4]. Maintenance then of proprioceptive mechanisms should play a role in the design of any splinting regimen undertaken at the elbow and other joints, and consideration should be given to research that suggests sensorimotor input and motor behaviour both change as soon as the cast, or immobilizing splint is applied [5]. Most interestingly, it has been noted that hand path alterations similar to those found in deafferented individuals were observed in subjects who had experienced electrophysical changes induced by twelve hours of upper limb immobilization. Clearly, when dealing with splints that immobilize the elbow joint to help manage the recovery of the joint after a bleed, a high premium must be placed on proprioceptive retraining once the period of range of motion restriction is passed. In addition, as the hemophilia population ages, some concern must be given to the risk of falling and the impact that preventing elbow joint motion might have on that level of risk. Research has shown that immobilization of the shoulder may have a negative effect on balance, and due to the impact that elbow immobilization has on movement higher up the kinetic chain at the shoulder joint, overall static and dynamic balance may be impaired and the risk of falling elevated if the elbow is devoid of movement [6]. An appreciation of this risk and the consideration of a falls assessment or falls prevention program may be warranted.

Whatever the goal of the splinting regimen, be it immobilization, structural support, or to allow for protected motion through a modified range of movement, consideration must be given to the convenience level of the device that is chosen. Up to $67 \%$ of patients required to wear an upper extremity splint on a continual basis report non-adherence with the splinting regimen [7], and so maximizing the convenience and comfort level of the splint is likely to impact the success of the treatment. To that end, one device that may function in several different capacities would be preferable. The use of a hinged, lockable elbow splint provides for a variety of applications throughout the acute, post-acute, and rehabilitative phases of recovery. With the hinge locked the brace becomes an effective joint immobilization device, customizable to the 
position of greatest comfort on an individual basis. For those patients who have full joint range of motion but require structural support to augment the function of the collateral ligaments, the hinge may be unlocked to allow for unrestricted motion within the splint's superstructure, guiding movement through a consistent pattern and providing enhanced lateral support.

Frequently the desired application is somewhere between these two extremes, as in the case of an elbow that exhibits chronic synovitis easily irritated by rapid or forced extension of the joint. The ability to lock the splint's hinge such that motion is restricted only as the joint approaches the potentially problematic position of terminal extension, allows for minimized functional loss by maintaining a mobile elbow. This also creates an environment amenable to proprioceptive retraining as the individual learns to actively control the movement towards end range with a reduced likelihood of developing a bleed. Gradually, as the synovitis settles, the splint may be adjusted to allow more joint extension in an incremental manner. The splint itself is lightweight, may be worn overtop of clothing or against bare skin, and may be swiftly and easily adjusted for range of motion increases or decreases, as well as total immobilization of the joint. These features maximize the comfort and convenience of the device, and help to improve adherence to the splinting regimen.

\section{The surgical approach}

Fortunately the shoulder joint which comprises the scapular-humeral joint and the gliding of the scapula over the rib cage permits many patients with elbow problems to function in activities of daily living, in a compromised way. Without the opportunity of combining shoulder movements with elbow function the need for corrective surgery would be far more common. Various operations have been described in the use of elbow arthropathy associated with haemophilia. These operations include synovectomy, simple excision of the radial head combined with joint debridement, excision arthroplasty, arthrodesis, and silastic interposition arthroplasty (1, $8,9,10,11)$. Excision of the radial head combined with synovectomy has resulted in consistently good results with reduction in pain, an increased range of forearm rotation (but not necessarily flexion/extension) and a reduction in the frequency of joint bleeds. Ulna nerve neurolyses have been carried out either by incision of the fibrous attachments around the ulna canal or by anterior transposition of the nerve. In advanced cases however, replacement arthroplasty may become indicated if there is significant destruction of the joint. 


\section{Elbow replacement}

The actual incidence of joint replacement however is likely to be low given that Bajekal reported that $81 \%$ of haemophiliacs suffered recurrent elbow bleeds but reported a low incidence of total joint replacement in the same group(12).

Whilst total joint replacement has been well described for the hip, knee and shoulder in haemophilia there have been few reports concerning total elbow replacement. Most reports have been restricted to isolated case reports $(10,13,14,15)$. The first report by Luck and Casper (10) reviewed the 20-year results of a total of 168 surgical procedures carried out for haemophilic arthropathy but included only two total elbow replacements, one of which became infected. Kasten and Skinner (16) in their large series of total elbow replacement described only two cases of haemophilia, one primary and a further revision.

The largest published series to date comes from the Oxford group and concerned seven elbow replacements in five patients with severe haemophilia $A$ (17). All patients demonstrated excellent relief of pain and improvement of function. There was one failure due to infection in an immuno-compromised patient with HIV and hepatitis $C$. The patients were followed for a minimum of 25 months and implants varied from unconstrained (Kudo or Souter-Strathclyde) to the more constrained Coonrad-Morrey joint replacements. There were three major postoperative complications, one ulnar nerve palsy, one axillary vein thrombosis and one patient who developed late infection requiring excision arthroplasty. They felt that the results were excellent in the short to medium term and they concluded that total elbow replacement is both feasible and useful in patients with severe haemophilic arthropathy.

Kaminemi (18)from the Mayo Clinic reported their results in five patients who had total elbow replacement. The mean age was 39 years with a mean follow-up of 5.8 years. Two patients died of AIDS and another from chronic renal failure. There were three complications in the five patients, one of uncontrollable bleeding, one of deep infection and one case with persistent pain. Wang et al (19) from Melbourne reported a series of six patients with a follow up of 6.2 years. Their functional results were excellent in three cases, good in two and fair in one. However two elbows required revision at a mean of 4.1 years post-operatively. 
All series describe the surgery in relatively young patients and all have a relatively short followup period. The early results have been encouraging however there is a relatively high proportion of early failure requiring revision. Although some of the outcomes are relatively optimistic, with complication rates approaching one in three and many requiring revision in less than five years, is perhaps not acceptable.

Overall the evidence and experience suggest that total elbow replacements in patients with haemophilia is an operation not to be undertaken lightly and should be performed only in circumstances of debilitating elbow symptoms. However frequently under these conditions the bone stock tends to be poor, there is an inevitably higher failure rate and the options for subsequent salvage are very limited.

\section{Conclusion}

The complexity of the elbow joint and the impact that symptoms have on the quality of life of a person with haemophilia means that it is a frequent cause of referral to the physiotherapist and surgeon. This paper has outlined the common physiotherapy and surgical approaches. It is important that these approaches continue to be evaluated in both the short and long term in order to determine the most effective treatment for the symptomatic elbow. 


\section{REFERENCES}

1. Gilbert, M. S. and Glass, K. S. Hemophilic arthropathy of the elbow. Mt Sinai J Med 44, 389396. 1977.

2. Søjbjerg JO. The stiff elbow. Acta Orthop Scand. 1996 Dec;67(6):626-31.

3. Leong DJ, Li YH, Gu XI, Sun L, Zhou Z, Nasser P, Laudier DM, Iqbal J, Majeska RJ, Schaffler MB, Goldring MB, Cardoso L, Zaidi M, Sun HB. Physiological loading of joints prevents cartilage degradation through CITED2. FASEB J. 2011 Jan;25(1):182-91.Epub 2010 Sep 8.

4. Krüger $M$, Eggert $T$, Straube A. Joint angle variability in the time course of reaching movements. Clin Neurophysiol. 2011 Apr;122(4):759-66. Epub 2010 Oct 27.

5. Moisello C, Bove M, Huber R, Abbruzzese G, Battaglia F, Tononi G, Ghilardi MF. Short-term limb immobilization affects motor performance. J Mot Behav. 2008 Mar;40(2):165-76.

6. Coleman A, Clifft J. The effect of shoulder immobilization on balance in community-dwelling older adults. J Geriatr Phys Ther. 2010 Jul-Sep;33(3):118-21.

7. Sandford F, Barlow N, Lewis J. A study to examine patient adherence to wearing 24-hour forearm thermoplastic splints after tendon repairs. J Hand Ther. 2008 Jan-Mar;21(1):4452; quiz 53.

8. Kay, L., Stainsby, D., and Buzzard, B.: The role of synovectomy in the management of recurrent haemarthroses in hameophilia. Br. J. Haematol. 49:53-60, 1981.

9. Le Balc'h, T., Ebelin, T., and Laurian, Y.: Synovectomy of the elbow in young hemophilic patients. J. Bone Joint Surg. Am. 69 A:264-269, 1987.

10. Luck, J. V., Jr.: Surgical management of advanced hemophilic arthropathy. Prog. Clin. Biol. Res. 324:241-256, 1990.

11. Rodriguez-Merchan, E. C., Galindo, E., Magallon, M., Cage, J., and Villar, A.: Resection of the radial head and partial open synovectomy of the elbow in the young adult with haemophilia. Haemophilia. 1:262-266, 1995.

12. Bajekal, R. A., Phillips, A. M., and Ribbans, W. J.: Elbow arthropathy in haemophilia. J. Bone Joint Surg. Br. Supp 1:15, 1996.

13. Beeton, K., Rodriguez-Merchan, E. C., and Alltree, J.: Total joint arthroplasty in haemophilia. Haemophilia. 6:474-481, 2000.

14. Phillips, A. M., Ribbans, W. J., and Goddard, N. J.: Ipsilateral total shoulder and elbow prosthetic replacement in a patient with severe haemophilia B. Haemophilia. 1:270-273, 1995. 
15. Utukuri M and Goddard, N. J.: Haemophilic arthropathy of the elbow. Haemophilia.11:565570, 2005.

16. Kasten, M. D. and Skinner, H. B.: Total elbow arthroplasty. An 18-year experience. Clin. Orthop.177-188, 1993.

17. Chapman-Sheath, P. J., Giangrande, P., and Carr, A. J.: Arthroplasty of the elbow in haemophilia. J. Bone Joint Surg. Br. 85:1138-1140, 2003.

18. Kaminemi S, Adams RA, O'Driscoll SW, Morrey BF: Hemophilic of the elbow treated by total elbow replacement. A case series. J. Bone Joint Surg. Am. 89 A:584-589, 2004.

19. Wang K, Street A, Dowrick A and Liew S: Clinical outcomes and patient satisafction following total joint replacement in haemophilia - 23 year experience in knees, hips and elbows. Haemophilia. 18:86-93, 2012. 